\title{
Teamwork enables high level of early mobilization in critically ill patients
}

\author{
Cheryl Elizabeth Hickmann, Diego Castanares-Zapatero, Emilie Bialais, Jonathan Dugernier, Antoine Tordeur, \\ Lise Colmant, Xavier Wittebole, Giuseppe Tirone, Jean Roeseler and Pierre-François Laterre*
}

\begin{abstract}
Background: Early mobilization in critically ill patients has been shown to prevent bed-rest-associated morbidity. Reported reasons for not mobilizing patients, thereby excluding or delaying such intervention, are diverse and comprise safety considerations for high-risk critically ill patients with multiple organ support systems. This study sought to demonstrate that early mobilization performed within the first $24 \mathrm{~h}$ of ICU admission proves to be feasible and well tolerated in the vast majority of critically ill patients.
\end{abstract}

Results: General practice data were collected for 171 consecutive admissions to our ICU over a 2-month period according to a local, standardized, early mobilization protocol. The total period covered 731 patient-days, 22 (3\%) of which met our local exclusion criteria for mobilization. Of the remaining 709 patient-days, early mobilization was achieved on $86 \%$ of them, bed-to-chair transfer on $74 \%$, and at least one physical therapy session on $59 \%$. Median time interval from ICU admission to the first early mobilization activity was $19 \mathrm{~h}(\mathrm{IQR}=15-23)$. In patients on mechanical ventilation ( $51 \%$ ), accounting for $46 \%$ of patient-days, $35 \%$ were administered vasopressors and $11 \%$ continuous renal replacement therapy. Within this group, bed-to-chair transfer was achieved on $68 \%$ of patient-days and at least one early mobilization activity on $80 \%$. Limiting factors to start early mobilization included restricted staffing capacities, diagnostic or surgical procedures, patients' refusal, as well as severe hemodynamic instability. Hemodynamic parameters were rarely affected during mobilization, causing interruption in only $0.8 \%$ of all activities, primarily due to reversible hypotension or arrhythmia. In general, all activities were well tolerated, while patients were able to self-regulate their active early mobilization. Patients' subjective perception of physical therapy was reported to be enjoyable.

Conclusions: Mobilization within the first $24 \mathrm{~h}$ of ICU admission is achievable in the majority of critical ill patients, in spite of mechanical ventilation, vasopressor administration, or renal replacement therapy.

Keywords: Teamwork, Intensive care unit, Mechanical ventilation, Early mobilization, Physical therapy, Perception

\section{Background}

Early mobilization referring to initiating physical exercise or mobilization within the early illness phase is an increasingly common practice in intensive care units (ICU) [1]. Yet the definition of early mobilization is rather vague, as it encompasses a wide range of techniques practiced on different ICU populations [2, 3]. Nevertheless, early mobility interventions in critically ill patients

\footnotetext{
${ }^{*}$ Correspondence: pierre-francois.laterre@uclouvain.be Intensive Care Unit, Cliniques universitaires Saint-Luc, Université catholique de Louvain (UCL), Avenue Hippocrate 10, 1200 Brussels, Belgium
}

prove to be feasible and safe in preventing bed-rest-associated morbidity [4-6], while improving patients' physical function [7], psychological condition [8], and quality of life [9]. Mobilizing patients at an early time point has been associated with reduced health care costs [10], as such intervention decreases invasive mechanical ventilation (MV) duration, delirium [7, 11], and hospital length of stay [12]. Recent observations suggest that providing mobility as early as possible and extending it to weekends could further improve patient outcomes [13-15].

Reported reasons for not mobilizing patients vary widely and include mechanical ventilation [16], 
catecholamine infusion [17], impaired consciousness [16], poor functional status [7, 12], safety considerations [9], limited staff capacities, or lack of protocols [18-20]. Safety considerations are indeed crucial in order to prevent additional risks, yet several reported safety issues are instrumental in excluding or delaying intervention in critically ill patients on multiple support systems, whereby this group runs the greatest risk of developing neuromuscular abnormalities.

At the same time, communication [21] and muscular activity [7] remain possible by means of limiting sedation, in line with current recommendations. Nevertheless, there is a lack of data available reporting patients' perceptions in such settings.

In our experience, early mobilization is an integral part of standard care, requiring teamwork combined with either limited sedation or none at all. The primary objective of this study was to demonstrate that early mobilization is feasible in the vast majority of critically ill patients, independently of their severity assessed by the need of $\mathrm{MV}$, high $\mathrm{FiO}_{2}$, vasopressor doses, or renal replacement therapy (RRT). The secondary objectives included safety of early mobilization, early mobilization rate in MV according to hypoxemia severity and patients' perception. Preliminary data were reported in an Abstract book [22].

\section{Methods}

\section{Setting and patients}

This was an observational study performed in a tertiary, 14-bed, mixed ICU at Saint-Luc University Hospital. Data were collected from all consecutive patients either already hospitalized in or newly admitted to our ICU between December 1, 2014, and January 31, 2015. The Ethics Committee of the Cliniques universitaires SaintLuc, Brussels, Belgium, approved the study protocol. A waiver was obtained for written informed consent, given that the described interventions were considered to be part of standard care. Early unwanted effects of mobility, in addition to monitoring data, were anonymously recorded in accordance with Belgian and European law.

\section{Early mobilization and standard care}

In accordance with the literature, we define early mobilization as a series of progressive physical activities able to induce acute physiological responses (enhancing ventilation, central and peripheral circulation, muscle metabolism, and alertness) [23] and beginning within $24 \mathrm{~h}$ of ICU admission. Our early mobilization protocol includes a few prior contraindications (Fig. 1) [24], such as acute myocardial infarction, active bleeding, increased intracranial pressure with major instability, unstable pelvic fractures, and therapy withdrawal. Moreover, during the morning medical rounds, a multidisciplinary team (physicians, physical therapists, and nurses) evaluates each patient in order to identify limitations to early mobilization. These include low blood pressure despite increasing dose of vasopressors, severe hypoxemia requiring a rapid increase in $\mathrm{FiO}_{2}$ or prone position, seizures, and patients' refusal.

According to the routine procedure for basic treatment, ICU team first transfers patients out of their beds. The ensuing physical therapy sessions are then designed as passive, active, or manual resistance exercise; cycle ergometer or leg press training; standing; verticalization by means of a tilt table; standing and assisted walking [25]. Activities are selected depending on patients' consciousness; hemodynamic/respiratory stability, as perceived by the team; as well as patients' preferences and physical capabilities. The complete therapeutic regime included getting out of bed together with physical therapy sessions twice a day. The daily mobilization program is otherwise considered to be incomplete.

Physical therapists are present at the ICU from Monday to Friday (7:30 am-5:00 pm), and the senior physical therapist-to-patient ratio is $1: 14$. The ratio of physical therapy students to senior physical therapists is $2: 1$. Furthermore, one resident physical therapist is present in the hospital at all times in case of respiratory emergencies. The nurse to patient ratio is 1:1.6 from 7:30 am to 4:00 pm.

Our standard care program consists in limited sedative administration in order to keep patients dozy and calm (RASS score between -1 and +1 ), combined with appropriate analgesia. Our preferred mechanical ventilation mode is pressure support, irrespective of hypoxemia severity or ARDS, provided that the protective volume and pressures guidelines were adhered to [26]. Controlled ventilation modes are mainly restricted to patients undergoing prone position or very severe hypoxemia despite PEEP adjustment.

\section{Data collection}

All medical and monitoring data were collected on a routine basis using our software of choice (Qcare 4.6 Build 154/2, C3 Critical Care Company NV, Sint-MartensLatem, Belgium), with subsequent analysis performed by means of a data extraction tool. We extracted from our routine database: demographic characteristics, severity scores, monitoring data, early mobilization activities, reasons for not providing such therapy, as well as any adverse events. Predefined adverse events included death, cardiac or respiratory arrest, falls, medical device removal, and abnormal physiological responses requiring activity interruption [27].

For the first patients' transfer to chair, the nurse monitored hemodynamic and respiratory parameters at 


\title{
Early mobilization protocol
}

\author{
M. Patri, CE. Hickmann, E. Bialais, J. Dugernier, P-F Laterre , J. Roeseler
} Intensive care unit, Saint Luc university hospital, Brussels.

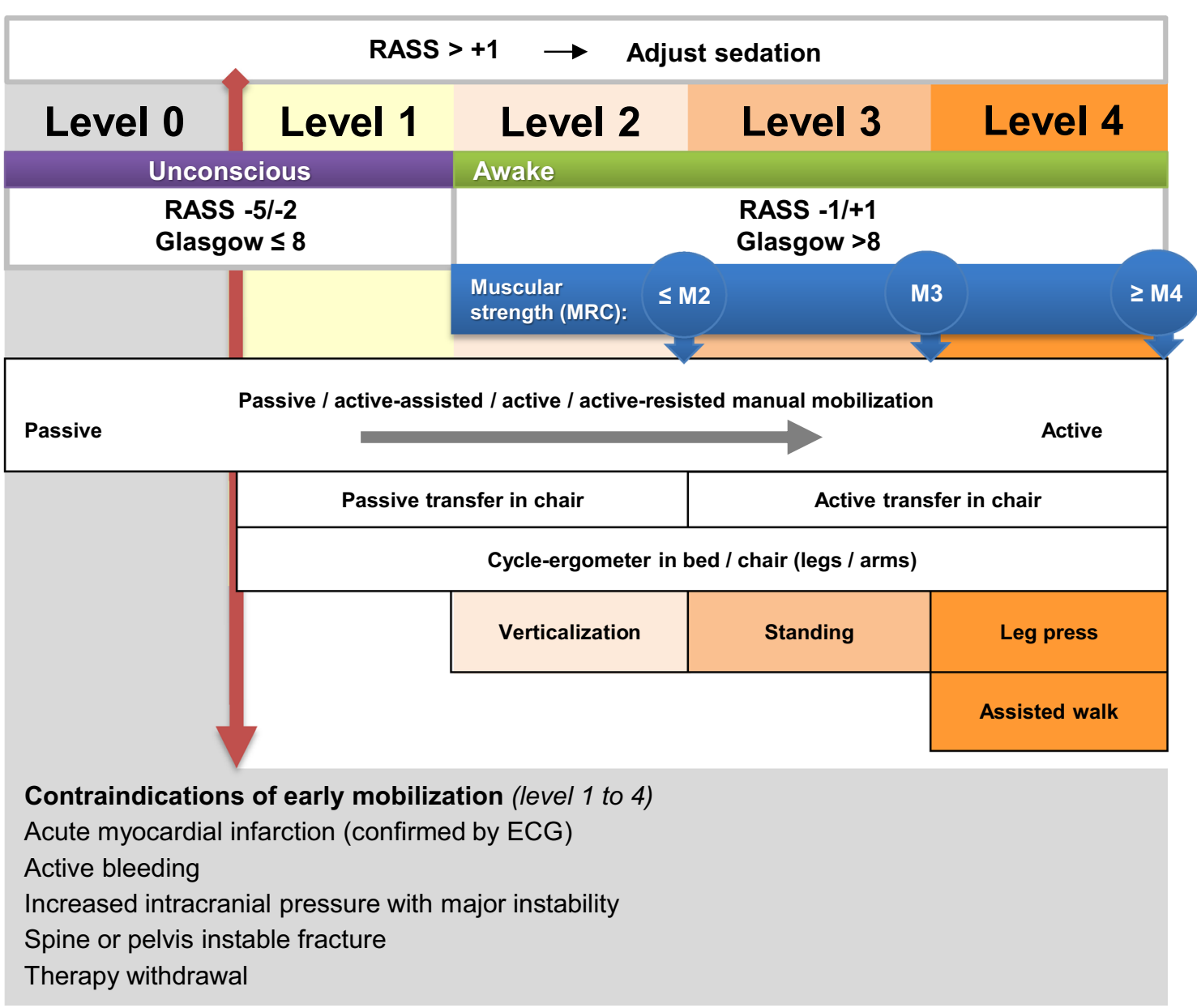

Fig. 1 Early mobilization protocol of ICU at Saint-Luc University Hospital. Modified with authorization [24]

baseline (in bed), and after 5 and $30 \mathrm{~min}$, respectively. Through physical therapy sessions, hemodynamic and respiratory parameters, along with pain scores, were monitored at baseline, as well as at 0 and 15 min afterward, respectively. Pain was assessed in communicative patients on a score of 0 (no pain) to 10 (maximum pain). Patients' perceived exertion was rated from 0 to 10 immediately following physical therapy sessions based on the Borg RPE scale [28], with a similar rating employed to measure perceived enjoyment $(0=$ no enjoyment, $10=$ maximum enjoyment $)[29,30]$.

\section{Statistical analysis}

Analyses were conducted using the software program SPSS software (IBM Corp. Released 2011. IBM SPSS Statistics for Windows, version 20.0. Armonk, NY, USA:
IBM Corp). Study periods were expressed in patient-days in terms of performing early mobilization therapy or lack thereof. Descriptive statistics were conducted for demographic, clinical, and activity data and expressed as mean and standard deviation or confidence interval at $95 \%$ (95\% CI) for normally distributed continuous variables, or as median and interquartile range (IQR) for non-normally distributed continuous variables. Categorical data were summarized using numbers or percentages. Characteristics between mobilized and non-mobilized patients were compared using unpaired Student's $t$ test or MannWhitney $U$ test when appropriate. Categorical data were compared with Chi-squared test between groups. Oneway repeated measures ANOVA was employed with time as a random factor in order to compare the effect of each activity on hemodynamic and respiratory parameters. 
To clearly demonstrate the safety of early mobilization, a multivariate analysis was performed by logistic regression. Adjusted odds ratio (AOR) for 28-day, ICU, and hospital mortality was calculated as follows: Univariate logistic regression analysis was previously performed to identify every numerical instability or collinearity of different factors associated with mortalities. Validated covariates were selected to be entered into a complete multivariate logistic regression model. Variable selection was performed with a method of backward elimination, using a criterion of $p$ value less than 0.20 for retention in the model. Final analysis was performed between covariates reaching a significant $p$ value. Statistical tests were two-sided, and significance was set at the 0.05 probability level.

\section{Results}

\section{Population description}

In total, 160 consecutive patients were admitted to the ICU over a 2-month period, and 11 others were already being hospitalized at the start of the study period. The overall characteristics of the 171 included patients are presented in Table 1. The mean APACHE II score was $18 \pm 7$ for the entire ICU population, $20 \pm 8$ for mechanically ventilated patients, and $22 \pm 7$ for those affected by severe sepsis or septic shock. Comorbidities were present in $60 \%$ of patients including; active cancer $(32 \%)$, end stage cirrhosis (14\%), neurologic disorders (9\%), chronic obstructive pulmonary disease (8\%), and pancreatitis (4\%). MV was provided to $51 \%$ of patients, including $14 \%$ with tracheostomy. Spontaneous modes, principally pressure support, were provided in $96 \%$ of days and controlled modes in only $4 \%$ of the mechanical ventilated population. Remaining patients had oxygenation by mask (13\%), high-flow oxygen therapy (6\%), noninvasive mechanical ventilation (1\%), or nasal cannula (21\%). The mean inspired oxygen fraction $\left(\mathrm{FiO}_{2}\right)$ in mechanically ventilated patients was $0.46 \pm 0.17$. Noradrenaline was the only vasopressor administered, with a mean dose of $0.16 \pm 0.23 \mu \mathrm{g} \mathrm{kg}^{-1} \mathrm{~min}^{-1}$. The primary sedatives employed were propofol (93\%) and clonidine (23\%). Neuromuscular blocking agents were only administered during tracheal intubation maneuvers, as necessary. Sedatives were administered to $84 \%$ of mechanically ventilated patients. The main analgesic medications, namely opioids and paracetamol, were administrated by means of intravenous bolus, patient-controlled analgesia systems, epidural, or oral route.

\section{Early mobilization therapy}

Overall, 139 (81 \%) patients underwent early mobilization therapy. The median (IQR) delay from ICU admission to patients' first activity was $19 \mathrm{~h} \mathrm{[15-23].} \mathrm{Seating} \mathrm{in}$ a chair was the first activity for $79 \%$ of patients. In these patients, proportion of hypoxemia according to Berlin classification [31] was as follows: without $(n=33)$, mild $(n=19)$, moderate $(n=40)$, and severe $(n=19)$. The 171 ICU admissions translated to 731 patient-days. Subjects displayed protocol exclusion criteria on $3 \%$ of patient-days. Reasons for this included active bleeding $(n=7)$, increased intracranial pressure with major instability $(n=3)$, unstable pelvic fractures $(n=2)$, and therapy withdrawal $(n=10)$. The remaining 709 were considered to be patient-days on which early mobilization was possible, thus accounting for 709 potential bedto-chair transfers and 1418 potential physical therapy sessions (Fig. 2), according to our protocol. Based on these totals, complete and partial mobility regimes were carried out on 48 and $86 \%$ of patient-days, respectively, and therefore incorporated into the treatment plan of $81 \%$ of admitted patients. Subjects were transferred from their beds to chairs on $74 \%$ of patient-days, with at least one physical therapy session provided on $59 \%$ of patient-days.

Mobilized and non-mobilized patients' characteristics are described in Table 2. MV, vasopressors, and RRT were provided on 46,30 , and $16 \%$ of patient-days, respectively. Patients treated using all the aforementioned support systems were transferred out of their beds on $60 \%$ of patient-days.

\section{Description of early mobilization}

Patients were transferred from bed to chair with assistance in standing upright in $60 \%$ of cases. They were manually lifted up by an ICU team in $36 \%$ of cases, with a motorized lift employed in the remaining $4 \%$. Patients remained in their chairs for a median (IQR) duration of 300 (152-300) min. Hemodynamic variations during the first sitting session did not differ between patients on mechanical ventilation and those without it (Additional file 1).

Active physical therapy sessions were provided to $61 \%$ of cases. Median (IQR) potency during active leg cycle ergometer sessions in seated and lying positions was recorded at 4 [3-5] watts and 3 [3-5] watts, respectively. Median (IQR) durations and RASS scores recorded during each activity are documented in Table 3.

The subjective perceptions of communicative patients were recorded on each physical therapy session (Table 3). Overall exertion ratings were moderate $(5 \pm 3)$; however, patients' enjoyment scores following physical therapy sessions were higher, indicating pleasant perceptions of their activity $(8 \pm 3)$, with even better values observed after more demanding activities, such as walking or active cycling. It is worth noting that pain was not significantly affected by physical activity. 
Table 1 Descriptive patient characteristics

\begin{tabular}{|c|c|c|c|}
\hline All admissions $(n=171)$ & $\begin{array}{l}\text { Mobilized } \\
n=139\end{array}$ & $\begin{array}{l}\text { Never mobilized } \\
n=32\end{array}$ & $p$ value \\
\hline $\mathrm{Age}^{\mathrm{a}}$ & $59 \pm 17$ & $62 \pm 17$ & 0.36 \\
\hline Male ${ }^{b}$ & $80(58 \%)$ & $18(56 \%)$ & 0.99 \\
\hline SOFA score ${ }^{a}$ & $5 \pm 3$ & $8 \pm 5$ & 0.01 \\
\hline APACHE II score ${ }^{a}$ & $17 \pm 7$ & $22 \pm 9$ & $<0.001$ \\
\hline $\begin{array}{l}\text { Predicted mortality (APACHE } \\
\text { II) }\end{array}$ & $29 \%$ & $44 \%$ & 0.017 \\
\hline In-hospital mortality ${ }^{\mathrm{b}}$ & $26(19 \%)$ & $16(50 \%)$ & $<0.001$ \\
\hline In ICU mortality ${ }^{b}$ & $11(8 \%)$ & $13(41 \%)$ & $<0.001$ \\
\hline 28-day mortality ${ }^{\mathrm{b}}$ & $15(11 \%)$ & $15(47 \%)$ & $<0.001$ \\
\hline ICU length of stay ${ }^{a}$ & $6.4 \pm 11.7$ & $1.4 \pm 2.1$ & 0.017 \\
\hline Vasoactive drug use $\mathrm{b}^{\mathrm{b}}$ & $47(34 \%)$ & $11(34 \%)$ & 0.99 \\
\hline Sedative drug use $\mathrm{b}^{\mathrm{b}}$ & $68(49 \%)$ & $13(41 \%)$ & 0.43 \\
\hline Opioids use $\mathrm{e}^{\mathrm{b}}$ & $86(62 \%)$ & $15(47 \%)$ & 0.16 \\
\hline Renal replacement therapy ${ }^{b}$ & $12(9 \%)$ & $5(16 \%)$ & 0.32 \\
\hline \multicolumn{4}{|l|}{ Admission cause } \\
\hline Medical $^{b}$ & $74(53 \%)$ & $15(47 \%)$ & 0.56 \\
\hline Elective surgery ${ }^{b}$ & $49(35 \%)$ & $9(28 \%)$ & 0.54 \\
\hline Urgent surgery $^{b}$ & $16(12 \%)$ & $8(25 \%)$ & 0.08 \\
\hline $\begin{array}{l}\text { Mechanically ventilated } \\
\text { patients }(n=88)\end{array}$ & $\begin{array}{l}\text { Mobilized } \\
n=69\end{array}$ & $\begin{array}{l}\text { Never mobilized } \\
n=19\end{array}$ & $p$ value \\
\hline $\mathrm{Age}^{\mathrm{a}}$ & $61 \pm 16$ & $66 \pm 14$ & 0.24 \\
\hline Male ${ }^{b}$ & $40(58 \%)$ & $12(63 \%)$ & 0.79 \\
\hline SOFA score ${ }^{a}$ & $7 \pm 4$ & $10 \pm 5$ & 0.01 \\
\hline APACHE II score ${ }^{a}$ & $19 \pm 7$ & $25 \pm 9$ & 0.005 \\
\hline $\begin{array}{l}\text { Predicted mortality (APACHE } \\
\text { II) }\end{array}$ & $36 \%$ & $60 \%$ & 0.003 \\
\hline In-hospital mortality ${ }^{\mathrm{b}}$ & $20(29 \%)$ & $13(68 \%)$ & 0.002 \\
\hline In ICU mortality ${ }^{b}$ & $11(16 \%)$ & $12(63 \%)$ & $<0.001$ \\
\hline 28-day mortality ${ }^{\mathrm{b}}$ & $10(14 \%)$ & $13(68 \%)$ & $<0.001$ \\
\hline ICU length of stay (days) ${ }^{a}$ & $10.7 \pm 15.5$ & $1.7 \pm 2.6$ & $<0.001$ \\
\hline MV duration (days) ${ }^{a}$ & $4.9 \pm 7.7$ & $1.3 \pm 1.1$ & 0.04 \\
\hline Vasoactive drug use $\mathrm{b}^{\mathrm{b}}$ & $39(57 \%)$ & $10(53 \%)$ & 0.79 \\
\hline Sedative drug use ${ }^{b}$ & $58(84 \%)$ & $13(68 \%)$ & 0.18 \\
\hline Opioids use $\mathrm{e}^{\mathrm{b}}$ & $47(68 \%)$ & $9(47 \%)$ & 0.18 \\
\hline Renal replacement therapy ${ }^{b}$ & $10(14 \%)$ & $5(26 \%)$ & 0.30 \\
\hline \multicolumn{4}{|l|}{$\mathrm{PaO}_{2} / \mathrm{FiO}_{2}$ ratio $^{\mathrm{b}}$} \\
\hline$>300(n=11)$ & $10(91 \%)$ & $1(9 \%)$ & 0.44 \\
\hline $201-300$ (mild) $(n=13)$ & $9(69 \%)$ & $4(31 \%)$ & 0.46 \\
\hline $\begin{array}{l}101-200 \text { (moderate) } \\
\quad(n=42)\end{array}$ & $34(81 \%)$ & $8(19 \%)$ & 0.61 \\
\hline$\leq 100$ (severe) $(n=22)$ & $16(73 \%)$ & $6(27 \%)$ & 0.55 \\
\hline $\begin{array}{l}\text { Non-mechanically } \\
\text { ventilated }(n=83)\end{array}$ & $\begin{array}{l}\text { Mobilized } \\
n=70\end{array}$ & $\begin{array}{l}\text { Never mobilized } \\
n=13\end{array}$ & $p$ value \\
\hline $\mathrm{Age}^{\mathrm{a}}$ & $56 \pm 17$ & $56 \pm 20$ & 0.96 \\
\hline Male ${ }^{b}$ & 40 (57\%) & $6(46 \%)$ & 0.54 \\
\hline SOFA score ${ }^{a}$ & $4 \pm 3$ & $5 \pm 5$ & 0.56 \\
\hline APACHE $\|$ score $^{a}$ & $15 \pm 6$ & $16 \pm 8$ & 0.67 \\
\hline $\begin{array}{l}\text { Predicted mortality (APACHE } \\
\text { II) }\end{array}$ & $22 \%$ & $19 \%$ & 0.69 \\
\hline
\end{tabular}

Table 1 continued

\begin{tabular}{|c|c|c|c|}
\hline $\begin{array}{l}\text { Non-mechanically } \\
\text { ventilated }(n=83)\end{array}$ & $\begin{array}{l}\text { Mobilized } \\
n=70\end{array}$ & $\begin{array}{l}\text { Never mobilized } \\
n=13\end{array}$ & $p$ value \\
\hline In-hospital mortality ${ }^{b}$ & $6(8 \%)$ & $3(23 \%)$ & 0.14 \\
\hline In ICU mortality ${ }^{b}$ & $0(0 \%)$ & $1(8 \%)$ & 0.15 \\
\hline 28-day mortality ${ }^{b}$ & $5(7 \%)$ & $2(15 \%)$ & 0.30 \\
\hline ICU length of stay ${ }^{a}$ & $2.2 \pm 1.6$ & $0.8 \pm 0.5$ & $<0.001$ \\
\hline Vasoactive drug use $^{b}$ & $8(11 \%)$ & $1(8 \%)$ & 0.99 \\
\hline Sedative drug use ${ }^{b}$ & $10(14 \%)$ & $0(0 \%)$ & 0.34 \\
\hline Opioids use ${ }^{b}$ & $39(56 \%)$ & $6(46 \%)$ & 0.55 \\
\hline Renal replacement therapy ${ }^{\mathrm{b}}$ & $2(3 \%)$ & $0(0 \%)$ & 0.99 \\
\hline \multicolumn{4}{|l|}{$\mathrm{PaO}_{2} / \mathrm{FiO}_{2}$ ratio $^{b}$} \\
\hline$>300(n=37)$ & $29(78 \%)$ & $8(22 \%)$ & 0.22 \\
\hline $201-300$ (mild) $(n=22)$ & $19(86 \%)$ & $3(14 \%)$ & 0.99 \\
\hline $\begin{array}{l}101-200 \text { (moderate) } \\
\quad(n=16)\end{array}$ & $15(94 \%)$ & $1(6 \%)$ & 0.44 \\
\hline$\leq 100$ (severe) $(n=8)$ & $7(88 \%)$ & $1(13 \%)$ & 0.99 \\
\hline \multicolumn{4}{|c|}{$\begin{array}{l}\text { APACHE II acute physiology and chronic health evaluation II score, SOFA } \\
\text { sequential organ failure assessment score }\end{array}$} \\
\hline \multicolumn{4}{|l|}{${ }^{\text {a }}$ Values expressed as mean $\pm S D$} \\
\hline \multicolumn{4}{|c|}{ b Values expressed as number (percentage) } \\
\hline
\end{tabular}

Hemodynamic parameters were recorded for 242 activities, 95 of which carried out by patients on MV while 147 involved no MV (Additional file 2). Heart rate, respiratory rate, or arterial pressure variations observed immediately after active exercises like walking, cycling, or manual mobilization were not clinically significant, returning to baseline values after $15 \mathrm{~min}$. Hemodynamic variations on active mobilization were similar for MV and non-MV patients.

\section{Limiting factors for mobilization activities}

Table 4 summarizes the limiting factors for early mobilization. ICU procedures (surgery, medical/nursing intervention, and imaging) were the most common reasons for patients not to perform mobilization activities, followed by physiological instability as perceived by the team, and then patients' refusal. The failure to provide any given physical therapy session was primarily accounted for by staff limitations on weekends, and the same applies to several physical therapist consultations during the week. To a lesser extent, mobilization activities were limited due to patients' refusal, ICU procedures, or physiological instability.

Hemodynamic instability was the most commonly reported physiological limitation to mobility, in patients receiving a mean dose of noradrenaline at $0.31(95 \% \mathrm{CI}$ $0.15-0.47) \mu \mathrm{g} \mathrm{kg}^{-1} \mathrm{~min}^{-1}$. Noradrenaline was administered during 361 mobilization activities at a mean dose of 0.10 (95\% CI 0.09-0.11) $\mu \mathrm{g} \mathrm{kg}^{-1} \mathrm{~min}^{-1}$. Active physical therapy was successfully performed for eight sessions, while the patients were on noradrenaline $>0.2 \mu \mathrm{g} \mathrm{kg}^{-1} \mathrm{~min}^{-1}$ [mean 


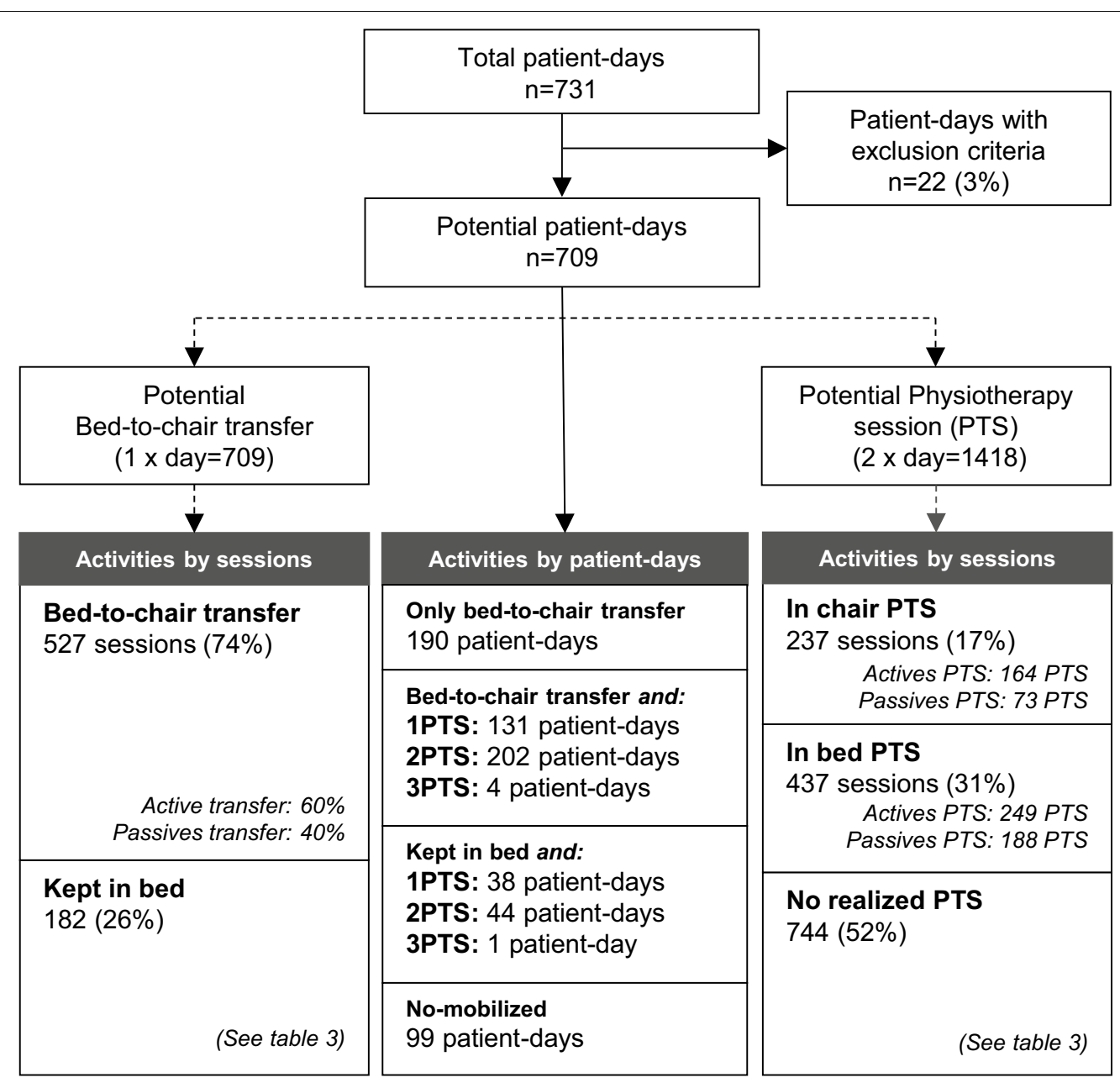

Fig. 2 Flowchart of early mobilization activities

dose: 0.34 (95 \% CI 0.11-0.44)] and transfer from bed to chair was performed for 11 sessions in the same condition [mean dose: 0.30 (95\% CI 0.22-0.37)].

The second limiting factor was related to respiratory dysfunction on account of recent intubation/extubation $(n=12)$, prone position $(n=2)$, or occurrence of severe hypoxemia $(n=19)$. In these patients, mean $\mathrm{FiO}_{2}$ was 0.62 (95\% CI 0.51-0.73). Nevertheless, $78 \%$ of MV patients were successfully mobilized with a mean $\mathrm{FiO}_{2}$ at 0.47 (95\%CI 0.46-0.49). We carried out 23 active and 49 passive physical therapy sessions with $\mathrm{FiO}_{2} \geq 0.60$ (mean $\mathrm{FiO}_{2}$ at 0.83 (95\%CI 0.77-0.88) and 0.71 (95\%CI 0.67-0.76), respectively), as well as 50 bed-to-chair transfers with mean $\mathrm{FiO}_{2}$ of 0.78 (95 \%CI 0.74-0.82). Maximum $\mathrm{FiO}_{2}$ at 1.0 was observed during 18 mobility activities: nine chair sittings and nine physiotherapy activities.

\section{Adverse events}

Activities were discontinued due to medical/nursing procedures in 11 cases and at patient request (pain, high perceived exertion, or digestive transit acceleration) in eight cases. Adverse events occurred in 10 interventions, representing $0.8 \%$ of total mobilizations; hypotension occurred in two patients receiving low-dose vasopressors, hypertension in two, and tachycardia in three. In the sitting position, one patient experienced faintness and was subsequently diagnosed with pulmonary embolism, while another epileptic patient experienced seizures. Moreover, one patient's operative wound exhibited slight oozing after a walking session. All events were reversible following activity interruption, displaying no impact on clinical outcome. There was no evidence of induced tissue hypoxia, as confirmed by means of steady lactate levels after mobilization available for 370 patients-days. 
Table 2 Characteristics of mobilized and non-mobilized patients

\begin{tabular}{|c|c|c|c|c|c|c|}
\hline & \multirow[t]{3}{*}{ ICU patient-days } & \multicolumn{4}{|l|}{ EM performed } & \multirow[t]{3}{*}{ No EM performed } \\
\hline & & \multicolumn{3}{|l|}{ Sitting in chair } & \multirow[t]{2}{*}{ In bed PTS+ } & \\
\hline & & All sitting in chair & PTS+ & PTS- & & \\
\hline Total & 709 & 527 & 337 & 190 & 83 & 99 \\
\hline Invasive mechanical ventilation (MV) & 327 & $223(68 \%)$ & $142(43 \%)$ & $81(25 \%)$ & $40(12 \%)$ & $64(20 \%)$ \\
\hline Severe sepsis/sepsis shock & 241 & $166(69 \%)$ & $102(42 \%)$ & $64(27 \%)$ & $28(12 \%)$ & $47(20 \%)$ \\
\hline Vasoactive drugs (VAD) & 211 & 149 (71 \%) & $99(47 \%)$ & $50(24 \%)$ & $25(12 \%)$ & $37(18 \%)$ \\
\hline Renal replacement therapy (RRT) & 115 & $76(66 \%)$ & $59(51 \%)$ & $17(15 \%)$ & $11(10 \%)$ & $28(24 \%)$ \\
\hline Sedatives (SD) & 260 & $193(74 \%)$ & $122(47 \%)$ & $71(27 \%)$ & $22(8 \%)$ & $45(17 \%)$ \\
\hline$M V+V A D$ & 158 & $104(66 \%)$ & $72(46 \%)$ & $32(20 \%)$ & $21(13 \%)$ & $33(21 \%)$ \\
\hline $\mathrm{MV}+\mathrm{VAD}+\mathrm{RRT}$ & 77 & $46(60 \%)$ & $38(49 \%)$ & $8(10 \%)$ & $8(10 \%)$ & $23(30 \%)$ \\
\hline MV + without SD & 122 & 77 (63 \%) & 49 (40 \%) & $28(23 \%)$ & $22(18 \%)$ & $23(19 \%)$ \\
\hline RASS -1 to +1 & 576 & 454 (79 \%) & 284 (49\%) & 170 (30\%) & $58(10 \%)$ & $64(11 \%)$ \\
\hline RASS $>+1$ & 25 & 21 (84 \%) & 18 (72 \%) & $3(12 \%)$ & $1(0.4 \%)$ & $3(12 \%)$ \\
\hline RASS $<-1$ & 108 & $50(46 \%)$ & 33 (31 \%) & $17(16 \%)$ & $22(20 \%)$ & 36 (33 \%) \\
\hline
\end{tabular}

Values expressed as number (percentage)

$M V$ mechanical ventilation, $V A D$ vasoactive drugs, $R R T$ renal replacement therapy, SD sedatives drug, RASS Richmond agitation-sedation scale, $P T S+$ physical therapy session carried out, PTS - no physical therapy session carried out, EM early mobilization

Table 3 Early mobilization activities and patients' perception

\begin{tabular}{|c|c|c|c|c|c|c|c|c|c|c|}
\hline & \multirow{3}{*}{$\begin{array}{l}\text { Total } \\
n\end{array}$} & \multirow{3}{*}{$\begin{array}{l}\text { Duration }{ }^{\mathbf{a}} \\
\text { min }\end{array}$} & \multirow{3}{*}{$\begin{array}{l}\text { RASS }^{a} \\
(-5 \text { to }+4)\end{array}$} & \multicolumn{7}{|c|}{ Patient perception $(0-10)^{b}$} \\
\hline & & & & \multicolumn{4}{|c|}{ Pain } & \multirow[t]{2}{*}{$n$} & \multirow{2}{*}{$\begin{array}{l}\text { Fatigue } \\
0 \mathrm{~min}\end{array}$} & \multirow{2}{*}{$\begin{array}{l}\text { Enjoyment } \\
0 \mathrm{~min}\end{array}$} \\
\hline & & & & $n$ & Before & $0 \mathrm{~min}$ & $15 \mathrm{~min}$ & & & \\
\hline In-bed passive mobilization & 151 & $17[15-20]$ & $-2[-4$ to 0$]$ & 11 & $4 \pm 3$ & $3 \pm 3$ & $3 \pm 3$ & 11 & $6 \pm 3$ & $8 \pm 1$ \\
\hline In-bed active mobilization & 177 & $18[15-22]$ & $0[0$ to 0$]$ & 121 & $4 \pm 3$ & $4 \pm 3$ & $4 \pm 3$ & 108 & $6 \pm 3$ & $7 \pm 3$ \\
\hline In-bed passive cycling (legs/arms) & 37 & $20[15-21]$ & $-1[-4$ to 0$]$ & 7 & $2 \pm 3$ & $2 \pm 3$ & $2 \pm 3$ & 7 & $5 \pm 3$ & $8 \pm 2$ \\
\hline In-bed active cycling (legs/arms) & 69 & $20[15-22]$ & $0[0$ to 0$]$ & 64 & $2 \pm 2$ & $2 \pm 2$ & $3 \pm 2$ & 65 & $5 \pm 3$ & $9 \pm 2$ \\
\hline In-bed leg press & 3 & $16[10-20]$ & $0[0$ to 0$]$ & 3 & $3 \pm 1$ & $3 \pm 1$ & $3 \pm 1$ & 3 & $5 \pm 1$ & $9 \pm 1$ \\
\hline In-chair sitting & 526 & $300[152-300]$ & $0[0$ to 0$]$ & - & - & - & - & - & - & - \\
\hline In-chair passive mobilization & 14 & $15[12-18]$ & $-2[-5$ to 0$]$ & 3 & $4 \pm 4$ & $4 \pm 4$ & $5 \pm 5$ & 1 & 3 & 5 \\
\hline In-chair active mobilization & 41 & $15[13-20]$ & $0[0$ to 0$]$ & 22 & $4 \pm 3$ & $4 \pm 3$ & $4 \pm 3$ & 16 & $6 \pm 2$ & $6 \pm 3$ \\
\hline In-chair passive cycling (legs/arms) & 59 & $20[15-20]$ & $0[-1$ to 0$]$ & 9 & $3 \pm 3$ & $4 \pm 3$ & $3 \pm 3$ & 4 & $4 \pm 1$ & $5 \pm 1$ \\
\hline In-chair active cycling (legs/arms) & 93 & $20[15-20]$ & $0[0$ to 0$]$ & 74 & $4 \pm 3$ & $4 \pm 3$ & $3 \pm 3$ & 65 & $5 \pm 3$ & $7 \pm 3$ \\
\hline In-chair leg press & 1 & 20 & 0 & 1 & 2 & 2 & 2 & - & - & - \\
\hline Standing/walking & 29 & 28 [20-40] & $0[0$ to 0$]$ & 24 & $2 \pm 2$ & $3 \pm 3$ & $3 \pm 2$ & 23 & $3 \pm 2$ & $9 \pm 2$ \\
\hline
\end{tabular}

$n$ Patient-days

a Values expressed as median [IQR]

b Values expressed as mean $\pm \mathrm{SD}$

\section{Safety of early mobilization}

By multivariate analyses, we were able to assess several risk factors associated with in ICU, 28-day, and in-hospital mortality (Additional file 3). Interestingly, after adjustment for severity covariates, early mobilization was not associated with increased mortality and was identified as a significant protective factor in all multivariate models
(AOR (95 \% CI): 0.06 (0.01-0.29), $p=0.001 ; 0.13(0.04-$ $0.47), p=0.002$ and $0.31(0.11-0.91), p=0.03$ for ICU, 28-day, and in-hospital mortalities, respectively). Longer ICU length of stay, advanced age, severity of hypoxemia according to Berlin classification, and higher SOFA score were risk factors for ICU mortality. Vasoactive drug use and higher APACHE II score were risk factors for 28-day 


\begin{tabular}{|c|c|c|}
\hline & \multicolumn{2}{|l|}{ Limiting factors to } \\
\hline & Bed-to-chair transfer & Physical therapy sessions \\
\hline & 182 out of $709(26 \%)$ & 744 out of 1418 (52 \%) \\
\hline \multicolumn{3}{|l|}{ Patient-dependent limiting factors } \\
\hline Severe physiological instability & $42(23 \%)$ & $42(6 \%)$ \\
\hline Hemodynamic instability & 21 & 9 \\
\hline Respiratory instability & 5 & 27 \\
\hline Neurological instability & 16 & 6 \\
\hline Patient refusal & $26(14 \%)$ & $62(8 \%)$ \\
\hline \multicolumn{3}{|l|}{ Patient-independent limiting factor } \\
\hline ICU interventions & $45(25 \%)$ & $49(7 \%)$ \\
\hline Surgery (transferred to OR) & 16 & 16 \\
\hline Medical/imaging procedures & 17 & 22 \\
\hline Nurse procedures & 12 & 13 \\
\hline Insufficient staff (weekend) & $11(6 \%)$ & $396(53 \%)$ \\
\hline Insufficient staff (weekdays) & $0(0 \%)$ & $16(2 \%)$ \\
\hline No reported physical therapist consultation during week & - & 177 (24\%) \\
\hline Unspecified & 58 (32 \%) & $2(0 \%)$ \\
\hline
\end{tabular}

Values expressed as number (\%)

$O R$ operative room

mortality. Finally, tracheostomy and higher APACHE II score were identified as risk factors for hospital mortality.

\section{Discussion}

This observational study demonstrates the utility of teamwork in successfully carrying out early mobilization, as assessed on 171 consecutive critically ill patients. The study's main observation is that mobility was provided at least once in $81 \%$ of all patients within $24 \mathrm{~h}$ of ICU admission. Bed-to-chair transfer was achievable in the vast majority of ICU patient-days. As shown by our study data, a teamwork approach exhibited an excellent safety profile when initiated very early after ICU admission, even in patients on support by vasoactive agents, MV, or RRT. Safety of our early mobilization approach was confirmed through a multivariate analysis taking into account patients' severity. After adjustment, early mobilization was identified not only as safe, but as a significant protective factor.

Despite the growing body of evidence confirming the feasibility, safety, and improved outcome displayed by early mobilization, it still remains a nonstandard and uncommon practice in ICUs. Moreover, initiation times vary significantly in the literature, ranging from 1.5 to 2 days [7, 32] to several days after intubation [9], or even weeks after ICU admission [33, 34]. Furthermore, several reports describe rehabilitation initiation occurring only after ICU discharge due to a lack of physical therapists or mobility teams within the ICU in question $[35,36]$. In a large-scale multicenter cohort study on MV patients, mobility was achieved in only $16 \%$ of overall sessions, reporting intubation and sedation as the primary limiting factors. In this report, authors founded a high incidence of muscular weakness and associated with higher mortality [16]. Furthermore, no clear improvement in outcome has been reported when reinforcement of physical activity was provided only after patients' awakening [37].

Recent expert recommendations on safety criteria for early mobilization mentioned that vasopressor use [38, 39], endotracheal intubation, RRT [38], or even life support devices like ECMO [40] should not be considered as contraindications for active mobilization. Despite that, besides the study of Pohlman et al. [32] performing in-bed mobilization with maximal $\mathrm{FiO}_{2}$ at 1.0 and vasoactive drug, no study has explored the safety of very early mobilization in critically ill patients on multiple support systems. To date, there is no consensus regarding vasoactive doses or maximum $\mathrm{FiO}_{2}$, but $<0.60$ was considered safe for initiating active mobilization [38]. Some authors consider a maximum noradrenaline dose of $0.2 \mu \mathrm{g} \mathrm{kg}^{-1} \mathrm{~min}^{-1}$ and $\mathrm{FiO}_{2}<0.55$ or 0.60 to be safe $[9,38]$. In the protocol at hand, we made a conscious effort to predefine a few contraindications, in order to assess each patient's potential to undergo early activity. Our results demonstrate that mobilizing patients with 
higher vasopressor doses and $\mathrm{FiO}_{2}$ is achievable without increased risks. However, based on our data we are unable to propose theoretical limits to mobilization. Indeed, there is to our view no limiting $\mathrm{FiO}_{2}$ or vasopressor dose, but rather a stabilized patient's condition with all supports.

Adverse event rates were shown to vary across studies. Pohlman et al. [32] reported the feasibility of early physical therapy and occupational therapy in $90 \%$ of MV patients on life support devices combined with daily sedation interruption. In their study, the mean Apache II score was 20, and mobility was initiated within 1.5 days following intubation, with adverse events occurring in $16 \%$ of overall sessions. In line with other studies, we clearly showed that most patients receiving $\mathrm{MV}$ and supportive therapy can be mobilized very early, within the first day of ICU admission. Furthermore, such activities were rarely interrupted due to adverse events like hypotension or arrhythmia, while requiring no additional intervention nor causing adverse outcome. We also demonstrated that mobility activities can be performed by patients following major abdominal surgery, patient that are often excluded of clinical trials.

As previously described, providing early mobilization with a high degree of supportive care requires experienced and coordinated multidisciplinary teams [41]. This is a mandatory aspect to ensure patients' security during early mobilization implementation.

Our principal limiting factor for specific physical therapy activities stemmed from staffing capacities, resulting in $28 \%$ of overall weekend and $12 \%$ of weekday physical therapy activities not being performed. This likewise accounted for the low rate of walks, since emphasis was placed on less time-consuming therapies, such as ergometer cycling, in an attempt to mobilize every patient. Based on our data, we estimated the ideal ratio of senior physiotherapists to patients to be $1: 7$ (including on weekends) in order to achieve the optimal number of daily physical therapy activities. Furthermore, the vast majority of patients were able to be moved out of bed by the nursing team on weekends. This observation confirms that a teamwork- and protocol-driven approach is recommended in order to ensure maximum mobilization, even in the presence of a limited number of physical therapists [19]. Moreover, even if more staff is required to mobilize patients out of bed, seating patients in a chair seems to be more advantageous in the ability to achieve a greater angle of inclination and to remain in a more stable position, compared with semi-recumbent position on bed, with non-additional risks [42].
Deep sedation is usually associated with limited mobility [43]. In our study, it was therefore unsurprising to observe a lower rate of bed-to-chair transfers for patients with a RASS score $<-1$. Current guidelines on sedation recommend maintaining consciousness with adequate analgesia, which results in a reduction in MV duration [44], vasopressor dosage, and in-hospital mortality [45]. In line with this recommendation, RASS scores in our study primarily ranged between -1 and +1 , allowing patients to communicate and self-regulate both exercise intensity and duration. In addition, patients were also allowed to refuse mobilization initiation, when expressing their inability to leave their beds or perform any physical activity. This overall approach therefore represents our optimal strategy to individually dose activity intensity and duration, coupled with vital parameter monitoring. In terms of severely ill unconscious patients, passive mobility has previously been reported to be associated with negligible variation in oxygen consumption and hemodynamic parameters [46-48].

Emerging clinical research now takes into consideration the subjective feelings of critically ill patients undergoing physical therapy in order to better dose their activities' intensity [49]. In accordance with such methods, overall exertion values in our population were moderate, coupled with higher perceptions of enjoyment post-exercise. These observations are highly relevant for this new approach of patient-centered outcomes in critical care. Surprisingly, even during the more demanding physical activities, patients reported high enjoyment ratings.

Our study has some limitations. Firstly, this was a single-center study conducted in an ICU with a strong culture of both mobilization and minimal sedation. It may thus prove difficult to extrapolate our results to other centers. Secondly, in line with our observational study design, muscle strength or other functional outcomes were not assessed. Moreover, the protective effect of early mobilization has to be considered as an observation in our study cohort and must be confirmed by a randomized controlled trial. At last, due to the layout of the critical care units in our hospital, we did not include ischemic or heart failure patients in our study.

In conclusion, we observed that early mobilization is achievable and well tolerated in the vast majority of critically ill patients, despite commonly described contraindications such as $\mathrm{MV}$, vasopressor administration, and RRT. It is of great interest to note that patients reported very positive experiences and feelings of wellbeing following various modalities of physical therapy sessions. 


\section{Additional files}

Additional file 1. Physiological responses during first transfer out of bed. Values expressed as mean \pm standard deviation; * different from baseline, $\approx$ different from $5 \mathrm{~min}$.

Additional file 2. Physiological responses of physiotherapy session. Values expressed as mean \pm standard deviation; $\mathrm{IB}=\ln$ bed, $\mathrm{IC}=\ln$ chair, * different from baseline, $\approx$ different from 0 min.

Additional file 3. Multivariate analyses for risk factors associated with mortality. AOR: adjusted odd ratio; \#surgery: elective or urgent surgery; * p-value $<0.05$

\section{Abbreviations}

ICU: intensive care unit; MV: mechanical ventilation; RRT: renal replacement therapy; ECMO: extracorporeal membrane oxygenation; $\mathrm{FiO}_{2}$ : fraction of inspired oxygen; PTS: physiotherapy session; EM: early mobilization; AOR: adjusted odds ratio.

\section{Authors' contributions}

$\mathrm{CEH}, \mathrm{CD}, \mathrm{EB}, \mathrm{JD}, \mathrm{XW}, \mathrm{JR}$, and PFL contributed to the conception and design of the research; AT, LC, GT, EB, JD, and JR contributed to data collection; all authors contributed to data analysis and interpretation and drafting of the manuscript. Furthermore, they all were involved in critically revising the manuscript and agree to be held fully accountable for ensuring the integrity and accuracy of their work. All authors read and approved the final manuscript.

\section{Acknowledgements}

The authors would like to thank Dr. Jean-Louis Bachy for his fundamental IT support and Professor Annie Robert for her assistance in statistical analyses.

\section{Competing interests}

The authors declare that they have no competing interests.

Received: 15 April 2016 Accepted: 15 August 2016

Published online: 24 August 2016

\section{References}

1. Burns JR. Letter: early ambulation of patients requiring ventilatory assistance. CHEST J. 1975;68(4):608a.

2. Bailey PP, Miller RR 3rd, Clemmer TP. Culture of early mobility in mechanically ventilated patients. Crit Care Med. 2009;37(10 Suppl):S429-35.

3. Stiller K. Physiotherapy in intensive care: an updated systematic review. Chest. 2013;144(3):825-47.

4. Chambers MA, Moylan JS, Reid MB. Physical inactivity and muscle weakness in the critically ill. Crit Care Med. 2009;37(10 Suppl):S337-46.

5. Griffiths RD, Palmer TE, Helliwell T, et al. Effect of passive stretching on the wasting of muscle in the critically ill. Nutrition. 1995:11(5):428-32.

6. Weber-Carstens S, Schneider J, Wollersheim T, et al. Critical illness myopathy and GLUT4: significance of insulin and muscle contraction. Am J Respir Crit Care Med. 2013;187(4):387-96.

7. Schweickert WD, Pohlman MC, Pohlman AS, et al. Early physical and occupational therapy in mechanically ventilated, critically ill patients: a randomised controlled trial. Lancet. 2009;373(9678):1874-82.

8. Hopkins RO, Suchyta MR, Farrer TJ, et al. Improving post-intensive care unit neuropsychiatric outcomes: understanding cognitive effects of physical activity. Am J Respir Crit Care Med. 2012;186(12):1220-8.

9. Burtin C, Clerckx B, Robbeets C, et al. Early exercise in critically ill patients enhances short-term functional recovery. Crit Care Med. 2009;37(9):2499-505.

10. Lord RK, Mayhew CR, Korupolu R, et al. ICU early physical rehabilitation programs: financial modeling of cost savings. Crit Care Med. 2013;41(3):717-24.
11. Skrobik Y, Chanques $G$. The pain, agitation, and delirium practice guidelines for adult critically ill patients: a post-publication perspective. Ann Intensive Care. 2013;3(1):9.

12. Morris PE, Goad A, Thompson C, et al. Early intensive care unit mobility therapy in the treatment of acute respiratory failure. Crit Care Med. 2008;36(8):2238-43.

13. Calvo-Ayala $\mathrm{E}$, Khan BA, Farber $\mathrm{MO}$, et al. Interventions to improve the physical function of ICU survivors: a systematic review. Chest. 2013;144(5):1469-80.

14. Peiris C, Shields N, Brusco N, et al. Additional Saturday rehabilitation improves functional independence and quality of life and reduces length of stay: a randomized controlled trial. BMC Med. 2013;11(1):198.

15. Hakkennes S, Lindner C, Reid J. Implementing an inpatient rehabilitation Saturday service is associated with improved patient outcomes and facilitates patient flow across the health care continuum. Disabil Rehabil. 2015;37(8):721-7.

16. TEAM Study Investigators. Early mobilization and recovery in mechanically ventilated patients in the ICU: a bi-national, multi-centre, prospective cohort study. Crit Care 2015;19(1):81

17. Bailey P, Thomsen GE, Spuhler VJ, et al. Early activity is feasible and safe in respiratory failure patients. Crit Care Med. 2007;35(1):139-45.

18. Barber EA, Everard T, Holland AE, et al. Barriers and facilitators to early mobilisation in Intensive Care: a qualitative study. Aust Crit Care. 2015;28(4):177-82 (quiz 83).

19. Jolley SE, Regan-Baggs J, Dickson RP, et al. Medical intensive care unit clinician attitudes and perceived barriers towards early mobilization of critically ill patients: a cross-sectional survey study. BMC Anesthesiol. 2014;14:84

20. Bakhru RN, Wiebe DJ, McWilliams DJ, et al. An environmental scan for early mobilization practices in U.S. ICUs. Crit Care Med. 2015:43(11):2360-9.

21. Egerod I, Bergbom I, Lindahl B, et al. The patient experience of intensive care: a meta-synthesis of Nordic studies. Int J Nurs Stud. 2015;52(8):1354-61

22. Jaillette E, Girault C, Brunin G, et al. French Intensive Care Society, International congress-Réanimation 2016. Ann Intensive Care. 2016;6(1):1-236.

23. Gosselink R, Bott J, Johnson M, et al. Physiotherapy for adult patients with critical illness: recommendations of the European Respiratory Society and European Society of Intensive Care Medicine Task Force on Physiotherapy for Critically III Patients. Intensive Care Med. 2008;34(7):1 188-99.

24. Offenstadt G. Réanimation - Traité de référence en Médecine Intensive et Réanimation. 3ème édition ed. Paris: Elsevier; 2016.

25. Choi J, Tasota FJ, Hoffman LA. Mobility interventions to improve outcomes in patients undergoing prolonged mechanical ventilation: a review of the literature. Biol Res Nurs. 2008;10(1):21-33.

26. Ventilation with lower tidal volumes as compared with traditional tidal volumes for acute lung injury and the acute respiratory distress syndrome The acute respiratory distress syndrome network. N Engl J Med 2000:342(18):1301-8.

27. Lee H, Ko YJ, Suh GY, et al. Safety profile and feasibility of early physical therapy and mobility for critically ill patients in the medical intensive care unit: beginning experiences in Korea. J Crit Care. 2015;30(4):673-7.

28. Borg G, Ljunggren G, Ceci R. The increase of perceived exertion, aches and pain in the legs, heart rate and blood lactate during exercise on a bicycle ergometer. Eur J Appl Physiol Occup Physiol. 1985;54(4):343-9.

29. Baron B, Moullan F, Deruelle F, et al. The role of emotions on pacing strategies and performance in middle and long duration sport events. $\mathrm{Br}$ J Sports Med. 2011;45(6):511-7.

30. Kilpatrick M, Kraemer R, Bartholomew J, et al. Affective responses to exercise are dependent on intensity rather than total work. Med Sci Sports Exerc. 2007;39(8):1417-22.

31. Ranieri VM, Rubenfeld GD, Thompson BT, et al. Acute respiratory distress syndrome: the Berlin definition. JAMA. 2012;307(23):2526-33.

32. Pohlman MC, Schweickert WD, PohIman AS, et al. Feasibility of physical and occupational therapy beginning from initiation of mechanical ventilation. Crit Care Med. 2010;38(11):2089-94.

33. Chiang $L L$, Wang $L Y$, Wu $C P$, et al. Effects of physical training on functional status in patients with prolonged mechanical ventilation. Phys Ther. 2006:86(9):1271-81. 
34. Martin UJ, Hincapie L, Nimchuk M, et al. Impact of whole-body rehabilitation in patients receiving chronic mechanical ventilation. Crit Care Med. 2005;33(10):2259-65.

35. Thomsen GE, Snow GL, Rodriguez L, et al. Patients with respiratory failure increase ambulation after transfer to an intensive care unit where early activity is a priority. Crit Care Med. 2008;36(4):1119-24.

36. O'Connor ED, Walsham J. Should we mobilise critically ill patients? A review. Crit Care Resusc. 2009;11(4):290-300.

37. Moss M, Nordon-Craft A, Malone D, et al. A randomized trial of an intensive physical therapy program for patients with acute respiratory failure. Am J Respir Crit Care Med. 2016;193(10):1101-10.

38. Hodgson CL, Stiller K, Needham DM, et al. Expert consensus and recommendations on safety criteria for active mobilization of mechanically ventilated critically ill adults. Crit Care. 2014;18(6):658.

39. Roeseler J, Sottiaux T, Lemiale $V$, et al. Management of early mobilisation (including electrostimulation) in adult and pediatric patients in the intensive care unit. Réanimation. 2013;22(2):207-18.

40. Ko Y, Cho YH, Park YH, et al. Feasibility and safety of early physical therapy and active mobilization for patients on extracorporeal membrane oxygenation. ASAIO J. 2015;61(5):564-8.

41. Bassett RD, Vollman KM, Brandwene $L$, et al. Integrating a multidisciplinary mobility programme into intensive care practice (IMMPTP): a multicentre collaborative. Intensive Crit Care Nurs. 2012;28(2):88-97.
42. Thomas P, Paratz J, Lipman J. Seated and semi-recumbent positioning of the ventilated intensive care patient-effect on gas exchange, respiratory mechanics and hemodynamics. Heart Lung. 2014;43(2):105-11.

43. Leditschke IA, Green M, Irvine J, et al. What are the barriers to mobilizing intensive care patients? Cardiopulm Phys Ther J. 2012;23(1):26-9.

44. De Jonghe B, Bastuji-Garin S, Fangio $P$, et al. Sedation algorithm in critically ill patients without acute brain injury. Crit Care Med. 2005;33(1):120-7

45. Tanaka LM, Azevedo LC, Park M, et al. Early sedation and clinical outcomes of mechanically ventilated patients: a prospective multicenter cohort study. Crit Care. 2014;18(4):R156.

46. Hickmann CE, Roeseler J, Castanares-Zapatero D, et al. Energy expenditure in the critically ill performing early physical therapy. Intensive Care Med. 2014;40(4):548-55.

47. Pires-Neto RC, Kawaguchi YMF, Hirota AS, et al. Very early passive cycling exercise in mechanically ventilated critically ill patients: physiological and safety aspects—a case series. PLoS One. 2013;8(9):e74182.

48. Koch SM, Fogarty S, Signorino C, et al. Effect of passive range of motion on intracranial pressure in neurosurgical patients. J Crit Care. 1996;11(4):176-9.

49. Sottile PD, Nordon-Craft A, Malone D, et al. Patient and family perceptions of physical therapy in the medical intensive care unit. J Crit Care. 2015;30(5):891-5.

\section{Submit your manuscript to a SpringerOpen ${ }^{\circ}$ journal and benefit from:}

- Convenient online submission

- Rigorous peer review

- Immediate publication on acceptance

- Open access: articles freely available online

- High visibility within the field

- Retaining the copyright to your article 